
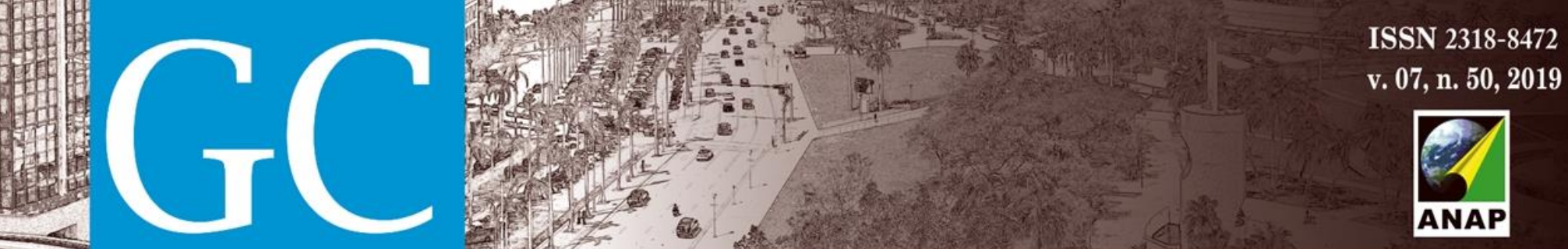

Revista Nacional de Gerenciamento de Cidades

\title{
Mapeando Intensidades Urbanas Com Uso de Ferramentas Digitais
}

\author{
Mapping urban intensities using digital tools
}

Mapeo de intensidades urbanas usando herramientas digitales

Caio Augusto Rabite de Almeida Doutorando, UFMG, Brasil. caio.rabite@gmail.com

Renato César Ferreira de Souza Professor Doutor, UFMG, Brasil. rcesarfs@gmail.com 


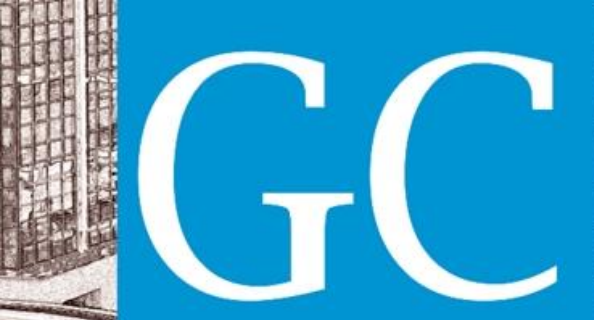

\section{Revista Nacional de} Gerenciamento de Cidades

território. Segundo Beirão (2005), independentemente da época ou das metodologias utilizadas, o desenho do espaço urbano é caracterizado como um processo complexo e com um longo período de concretização e reflexão.

As análises promovidas com a inserção de tecnologias digitaisnão apenas nos permitem capturar a influência da forma urbana, mas também nos informam como o planejamento e as decisões de projeto que moldam os futuros ambientes construídos podem ser operacionalizados para alcançar condições de cidades mais orientadas.

\section{METODOLOGIA}

O método proposto para o desenvolvimento deste trabalho foi a análise de conexões e a classificação das métricas espaciais da concentração de atividades de um determinado local, usando as ferramentas disponíveis através do plugin UNA Toolbox operando no software de modelagem Rhinoceros, que foi desenvolvido pelo grupo de pesquisa City Form Lab da Universidade de Harvard, nos Estados Unidos. A escolha por esta ferramenta, foi apoiada na possibilidade de inserir dados e operar em um ambiente digital para a mensuração das relações de intensidades urbanas presentes em uma determinada área.

As informações que são necessárias para a configuração das redes e posterior análise de intensidade, podem ser importadas para o Rhino a partir de diversas fontes existentes, como bancos de dados GIS, arquivos Open Street Map e mapas de base CAD, ou então rastreados diretamente no software pelos próprios usuários. As redes podem ser compostas de quaisquer elementos de curva (polilinhas, arcos, curvas splines) e formar redes bidimensionais e/ou tridimensionais. O Rhino permite criar e editar as redes de maneira bem flexível, por se tratar de um programa de modelagem de natureza NURBS (Non-Uniform Rational Basis-Spline) usada para representar curvas e superfícies com maior precisão e flexibilidade, conforme demonstrado na Figura 1. 


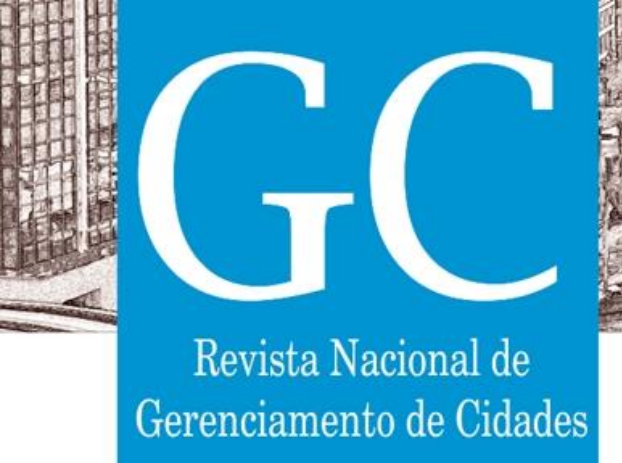

transporte. Após realizadas essas entradas de informações dadas pelo usuário, o passo seguinte é descrever quais as métricas e funções desejadas a serem exibidas, como por exemplo ao se medir a facilidade ou a influência com que as origens das redes espaciais podem acessar um conjunto dado de destinos, conforme demonstrado na Figura 2.

Figura 2: Descrição de algumas métricas utilizadas através do plugin UNA Toolbox.

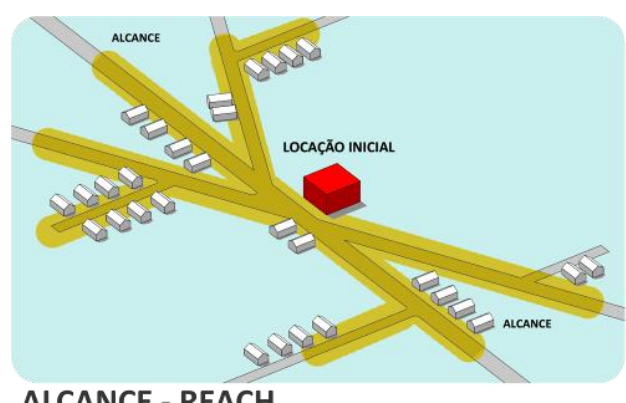

ALCANCE - REACH

Ao caminhar pelas ruas da cidade, quantos locais são acessíveis dentro de uma determinada distância a partir de um local de partida

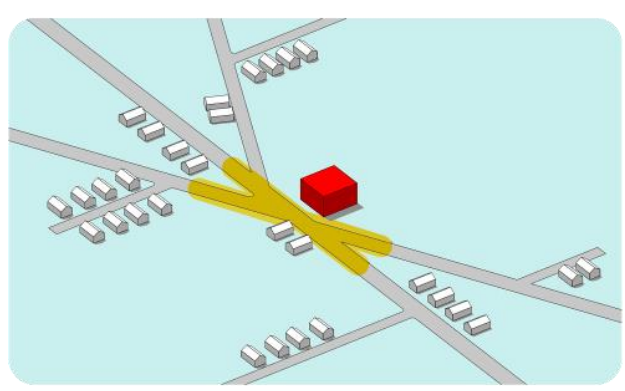

\section{CENTRALIDADE - BETWENESS}

Uma medida que pode ser usada para estimar a quantidade de tráfego a pé que um local recebe.

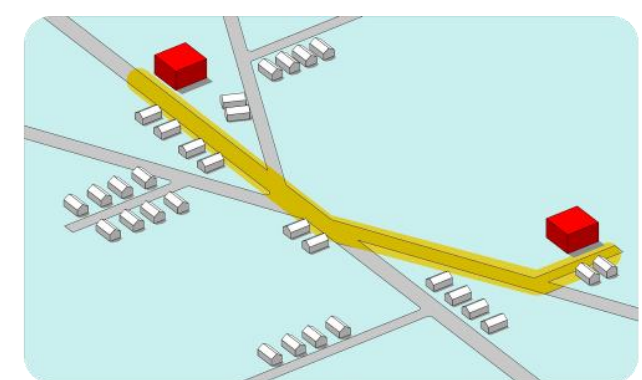

\section{LINEARIDADE - STRAIGHTNESS}

A extensão em que partes de um local para outros nós de interesse são linhas retas. Normalmente, as pessoas preferem rotas com poucas alternâncias.

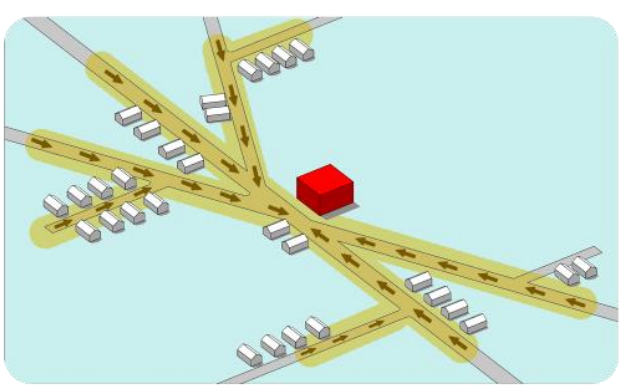

\section{PROXIMIDADE - CLOSENESS}

Uma medida de quão central é um ponto, medido pelos caminhos mais curtos para nós próximos, que ocorrem na interação das ruas.

Fonte: Elaboração Própria, 2019.

A métrica de alcance (Reach) quantifica quantos destinos cada origem pode alcançar dentro de um raio determinado. Esta funcionalidade pode ser usada para descrever quantos lares ou empregos estão disponíveis em uma caminhada de cinco minutos ao redor de cada ponto de ônibus ou comércio varejista por exemplo. A análise de proximidade (Closeness), pode ser usada para estimar o fluxo de pedestres em locais específicos. Como os pedestres nem sempre escolhem rotas mais curtas, o movimento entre cada origem e destino pode não apenas ser modelado ao longo dos caminhos mais curtos, mas os usuários também podem inserir uma "razão de desvio" permitida para incluir rotas mais longas. Se as casas são tomadas como origens e os varejistas como destinos, então o número de residentes a partir de cada local de residência é distribuído por todos os caminhos dentro do desvio permitido, dando a cada rota uma 


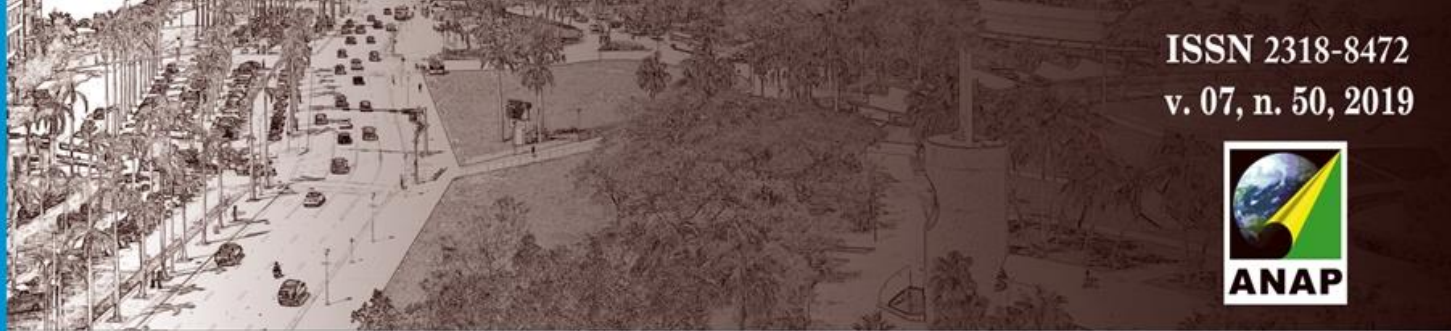

Revista Nacional de Gerenciamento de Cidades

probabilidade igual. A análise monitora quais segmentos de rota recebem a maioria dos usuários gerais, indicando o fluxo total de pedestres estimado.

As análises de mensuração de "Patrocínio" (Patronage) de instalações locais podem ser usadas para estimar o estímulo de instalações espaciais diversificadas - por exemplo, comércio, espaços públicos, estacionamentos - em uma rede. Um modelo de escolha discreta é usado para alocar uma proporção de demanda de todos os pontos de origem a todas as instalações de destino, de modo que uma alocação mais alta seja dada às instalações que estão mais próximas do usuário ou tenham pesos mais altos. Os resultados podem ser usados para examinar quantas pessoas ou que parte da demanda total é susceptível de apoiar cada instalação. Testar diferentes cenários de planejamento pode melhorar o favorecimento de instalações para destinos individuais ou todos os destinos coletivamente. Por fim, a opção da centralidade (Betweeness), pode ser utilizada para estimar a quantidade de tráfego de pedestres que uma área recebe. Essa abordagem poderia ser usada, para estimar o impacto que determinada nova edificação pode provocar em determinado lugar ou decidir quais os melhores pontos de impacto de transporte e acesso nessa área.

Após apontar dentro da rede quais os pontos de origem e destino e seus pesos dentro da mesma, bem como o tipo de análise a ser verificada pelo software, é gerado um gráfico de cores que pode ser editado graficamente pelo usuário, em que o padrão escolhido indica através de pontos aqueles de maior influência ou proximidade para determinada cor (Figura 3.), esses atributos também podem ser demonstrados numericamente para cada ponto.

\section{EXPERIMENTO}

Para uma experimentação da abordagem metodológica tratada por esta pesquisa, realizou-se um estudo de caso em uma área selecionada na cidade de Juiz de Fora, Brasil. O primeiro estágio do experimento foi a escolha de lugar da cidade em que a pesquisa foi realizada e a geração de sua malha urbana. Os critérios levaram em consideração o contraste da diversidade de usos e dos agentes envolvidos com o espaço e de sua consequente dinâmica social presente.

Para ilustrar o conceito de intensidade utilizada, é necessário fazer-se uma distinção entre densidade urbana e intensidade utilizada pela ferramenta de análise deste trabalho. Enquanto a densidade se refere à quantidade de pessoas ou elementos da forma urbana (por exemplo, unidades habitacionais, área útil) por unidade de área de terra, a intensidade refere-se à concentração de atividades comerciais e de serviço nos andares térreos ao longo das ruas da cidade. Chamamos essa intensidade de qualidade do piso térreo, já que descreve a interface do ambiente urbano que as pessoas se envolvem mais diretamente e encontram-se no dia a dia. A definição de intensidade parte da referência de volume de interações espaciais que o térreo de um determinado perímetro tem a oferecer, portanto as redes de rua que possuem maiores concentrações de atividades são consideradas mais intensas. Segundo Larice (2006), são as 


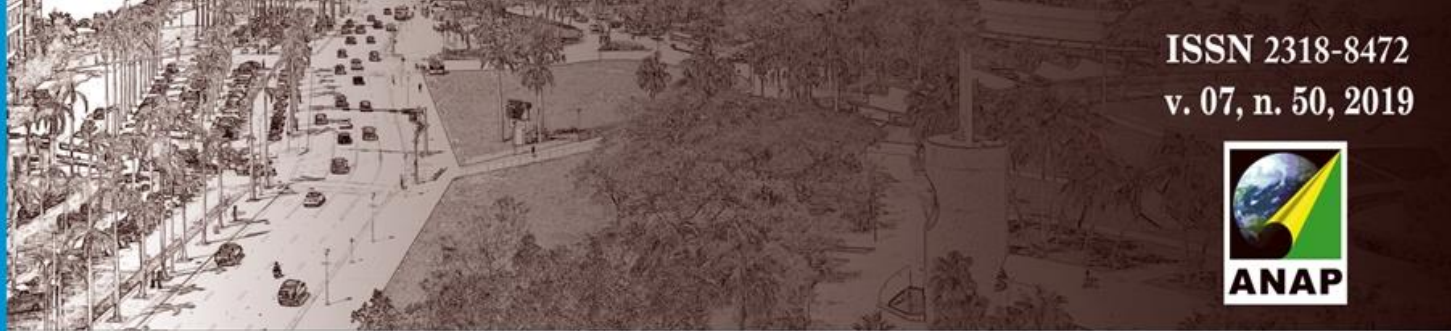

Revista Nacional de Gerenciamento de Cidades

Geográfica com teorias e práticas de urbanismo paramétrico, onde o foco é a coleta de dados, análise de padrões, visualização e modelagem espacial. Perceber esses comportamentos na estruturação e planejamento de uma cidade e de seus espaços, tornam-se instrumentos que podem ser utilizados para visualização e formação de estratégias de projeto.

\section{RESULTADOS}

A pesquisa permitiu não somente uma maior compreensão da complexidade e essência local dos ambientes urbanos com o reconhecimento da multidimensionalidade de suas provocações, mas também permitem identificar conexões e visualizar a dinâmica socioeconômica do(s) lugar(es). A comparação das informações obtidas permitiu reconhecer a relação entre espaço físico, redes estabelecidas e ações locais. Tal como apontou Rodriguez et al (2013), a tarefa de coleta de dados e mapeamento são de suma importância para revelar as propriedades do lugar. Se as forças do lugar forem identificadas e conceituadas nas etapas de avaliação e coleta com direcionamento desde o início, a arquitetura e o planejamento urbano podem ser propostos para atuar em consonância com estas forças.

Uma das proposições futuras seria a de compreender quais as contribuições que podem ser feitas por meio de uso de ferramentas paramétricas e construção de modelos efetivos de compensação do território e do uso local do espaço, ou ainda a distribuição do layout de serviços dentro de um território, com base nas necessidades reais dos usuários e superando padrões impostos por padrões de urbanismo e planos diretores para regulamentação qualitativa de espaço baseadas em mapas e estudos comparativos.

Com isto pode-se apontar que essas ferramentas podem possibilitar não somente uma melhor proposição de solução e entendimento de políticas de urbanização, mas também para a sociologia, meio ambiente, filosofia, economia e todos os vários agentes e interessados na complexidade da dinâmica das cidades latino-americanas. $O$ desafio torna-se em como fornecer aos gestores urbanos novas maneiras que permitam realizar análises e previsões em tempo hábil para servir de suporte na tomada de decisões mais proativas que permitam a um direcionamento de desenvolvimento mais eficiente e sustentável.

O desenvolvimento de ferramentas digitais tem permitido dessa forma um fluxo interno de dados dos quais tornou-se possível a análise de cidades de maneiras que não poderiam ser previamente antecipadas. Esses dados podem aplicados construtivamente em uma maneira que possa indicar formas de melhorar não somente padrões urbanos, mas também na vivência econômica e social. 


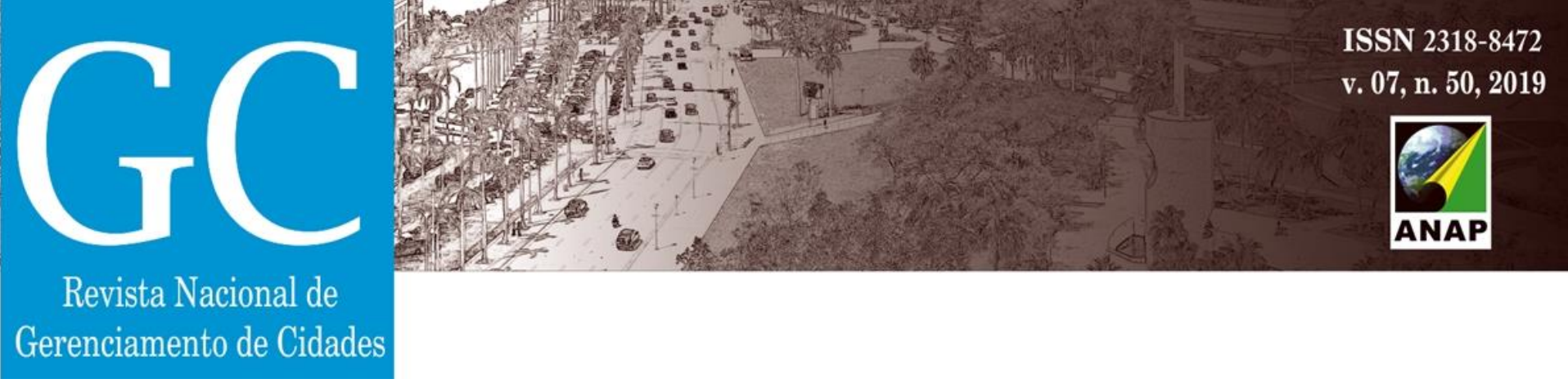

\section{DISCUSSÕES}

O local, sob a ótica de dados espaciais, pode ser um instrumento chave para a visualização de cenários atuais, previsão de impactos de transformação e aprimoramento de prestação de serviços. A informação sobre a localização pode ser capaz de possibilitar análises com grande complexidade na distribuição espacial de lugares, eventos e serviços, bem como fornecer oportunidades para vincular outras informações, interagir com usuários e otimizar as capacidades de feedback e contribuição do espaço.

Até o presente momento, a pesquisa aqui demonstrada tem focado de uma maneira mais explícita na morfologia urbana, mas é pertinente que se realizem pesquisas de acompanhamento, reunindo dados de atividades etnográficas sobre como as ruas e os espaços estão sendo utilizados e transformados, vinculando as evidências comportamentais aos padrões espaciais e econômicos.

Apesar dos desenvolvimentos tecnológicos e esforços feitos na criação e uso de ferramentas de sistema informação geográfica, de análise e planejamento urbano, o que se observa é que a maior parte destes recursos ainda se encontram em um estágio emergente, onde suas potencialidades ainda não são usadas de maneira plena ou em estágios muito prematuros de prática, principalmente tratando-se de um contexto sul americano de aplicação.

\section{REFERÊNCIAS BIBLIOGRÁFICAS}

ABRAMS, J; P. Hall. Else/where : mapping. Minneapolis, Minn, University Of Minnesota Design, 2006.

BATTY, M. Cities, Complexity, and Emergent Order. Publicação em complexity.info, 2011. Acesso em: 30 de Julho de 2019. http://www.complexcity.info/files/2011/09/BATTY-CITIES-ERA21.pdf

BEIRAO, J.; DUARTE, J. (2005) Urban Grammars: Towards Flexible Urban Design. Session 11: shape grammars - eCAADe 23, 2005.

GOODCHILD, M. Citizens as Sensors: the World of Volunteered Geography. GeoJournal, 69 (4): 211-221, 2007.

KOENIG, R.; MIAO, Y.; KNECHT, K.; BUŠ, P.; MEI-CHIH, C. Interactive Urban Synthesis. In Proceedings of the Computer-Aided Architectural Design: Future Trajectories: 17th International Conference, CAAD Futures 2017, Istanbul, Turkey, 10-14 July 2017; Volume 724, p. 23.

LARICE, M.A. Great neighborhoods: The livability and morphology of high density neighborhoods in urban North America. University of California, Berkeley, 2006.

MCNEEL, R. RHINOCEROS - NURBS MODELING FOR WINDOWS (VERSÃO 6.0), McNeel North America, Seattle, W A, USA. www.rhino3d.com/. Acesso em: 07 de agosto de 2019.

PORTAS, N. A Cidade como Arquitectura. Lisboa: Livros Horizonte, 2007.

RHINOCOMMON SDK, Official Website: http://www.rhino3d.com/5/rhinocommon/. Acesso em: 07 de agosto de 2019. 


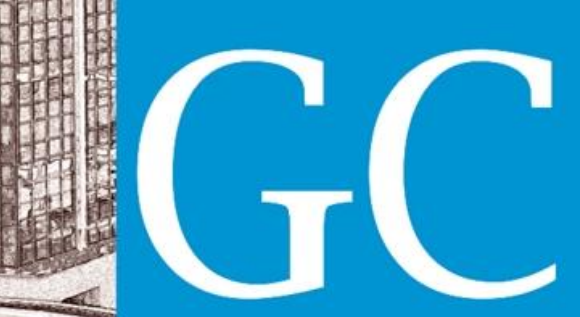

\section{Revista Nacional de} Gerenciamento de Cidades

RODRIGUEZ, A.; SANZA, P. Visualizing the (in)visible: mapping culture and the forces that shape the urban experience. In: SiGraDi - Knowledge Based-Design, 2012, Valparaíso, Chile, Anais da XVII Conference of the Iberoamerican Society of Digital Graphics, 2013, 95-99.

SEVTSUK, A.; MEKONNEN, M. Urban Network Analysis: A new toolbox for ArcGis, Revue international de géomatique 2, no.2: 287-305, 2012.

SEVTSUK, A.; NIXON F.; AMINDARBARI, R. Capturing Urban Intensity; In Open Systems: Proceedings of the 18th International Conference on Computer-Aided Architectural Design Research in Asia, 551-560. CAADRIA. Singapore: The Association for Computer-Aided Architectural Design Research in Asia, 2013.

UNA TOOLBOX, version 6.13.10.37 - Urban network Analysis for Rhinoceros 6 3D, Desenvolvido por City Form Lab - Harvard, Disponível em: https://www.food4rhino.com/app/urban-network-analysis-toolbox. 